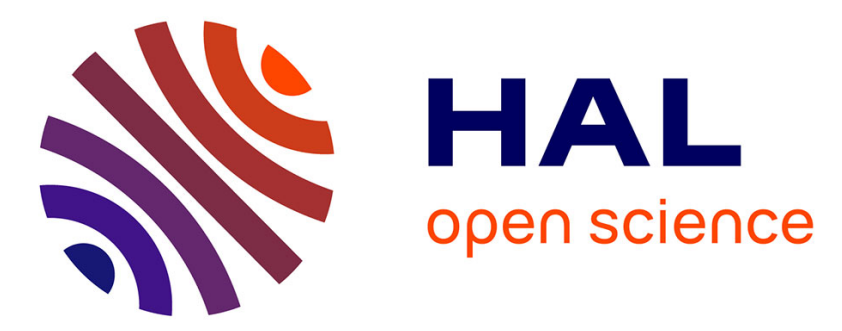

\title{
Diverses résonances de croisement de niveaux sur des atomes pompés optiquement en champ nul II. Applications à la mesure de champs faibles
} C. Cohen-Tannoudji, J. Dupont-Roc, S. Haroche, F. Laloë

\section{- To cite this version:}

C. Cohen-Tannoudji, J. Dupont-Roc, S. Haroche, F. Laloë. Diverses résonances de croisement de niveaux sur des atomes pompés optiquement en champ nul II. Applications à la mesure de champs faibles. Revue de Physique Appliquée, 1970, 5 (1), pp.102-108. 10.1051/rphysap:0197000501010200 . jpa-00243340

\section{HAL Id: jpa-00243340 https://hal.science/jpa-00243340}

Submitted on 1 Jan 1970

HAL is a multi-disciplinary open access archive for the deposit and dissemination of scientific research documents, whether they are published or not. The documents may come from teaching and research institutions in France or abroad, or from public or private research centers.
L'archive ouverte pluridisciplinaire HAL, est destinée au dépôt et à la diffusion de documents scientifiques de niveau recherche, publiés ou non, émanant des établissements d'enseignement et de recherche français ou étrangers, des laboratoires publics ou privés. 


\title{
DIVERSES RÉSONANGES DE GROISEMENT DE NIVEAUX SUR DES ATOMES POMPÉS OPTIQUEMENT EN GHAMP NUL \\ II. APPLIGATIONS A LA MESURE DE GHAMPS FAIBLES
}

\author{
Par C. COHEN-TANNOUDJI, J. DUPONT-ROG, S. HAROGHE et F. LALOË,
}

Faculté des Sciences, Laboratoire de Spectroscopie hertzienne de l'E.N.S., associé au C.N.R.S., 24, rue Lhomond, 75-Paris $\left(5^{\mathrm{e}}\right)$

Résumé. - On décrit la réalisation et les performances d'un magnétomètre utilisant les résonances de croisement de niveaux étudiées dans la communication précédente et observées sur une vapeur de ${ }^{87} \mathrm{Rb}$ pompée optiquement. La très grande finesse des résonances (de l'ordre de $1 \mu \mathrm{G}$ ) permet la détection de champs très faibles, de l'ordre de $10^{-9}$ à $10^{-10} \mathrm{G}$ avec une constante de temps de $3 \mathrm{~s}$ à la détection. On décrit également une expérience de détection à l'aide de ce magnétomètre du champ magnétique statique créé par des noyaux de ${ }^{3} \mathrm{He}$ orientés en phase gazeuse.

Abstract. - One describes the main characteristics of a magnetometer using the level crossing resonances studied in the previous communication and detected on optically pumped ${ }^{87} \mathrm{Rb}$ atoms. The extreme narrowness of these resonances (width of about $1 \mu \mathrm{G}$ ) allows the detection of very weak fields $\left(10^{-9}-10^{-10} \mathrm{G}\right)$ with a $3 \mathrm{~s}$ detection time constant. The detection, with this magnetometer, of the static magnetic field produced by oriented ${ }^{3} \mathrm{He}$ nuclei in a vapor is also reported.

Introduction. - Nous allons décrire la réalisation et les performances d'un magnétomètre utilisant les résonances étudiées dans la communication précédente et observées sur une vapeur de ${ }^{87} \mathrm{Rb}$ pompée optiquement [1]. Ce magnétomètre, exploitant la très grande finesse de ces résonances, permet de détecter des champs très faibles, de l'ordre de $10^{-9}$ à $10^{-10} \mathrm{G}$ avec une constante de temps de $3 \mathrm{~s}$ à la détection. Nous allons successivement indiquer le principe du magnétomètre $(\S \mathrm{A})$, en décrire les différentes parties $(\S \mathrm{B})$, indiquer les opérations de réglage qui accompagnent les mesures $(\S \mathrm{G})$ et discuter sa sensibilité $(\S \mathrm{D})$.

Une telle sensibilité rend intéressante l'application de ce magnétomètre à l'étude de nombreux phénomènes magnétiques dans le domaine des champs très faibles. Nous en donnerons dans cette communication un exemple se rapportant à l'étude des milieux très faiblement magnétiques en décrivant une expérience de détection du champ magnétique statique créé par des noyaux de ${ }^{3} \mathrm{He}$ orientés $(\S \mathrm{E})$.

A. Principe du magnétomètre. - Le schéma de principe du magnétomètre est indiqué sur la figure 1 : la cellule de résonance contenant les atomes de ${ }^{87} \mathrm{Rb}$,

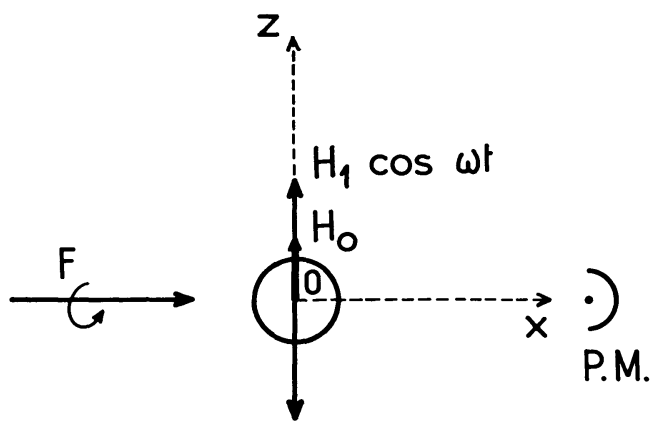

FIG. 1. centrée en 0 , est pompée par un faisceau lumineux $\mathrm{F}$, polarisé circulairement et dirigé le long de $O x$ perpen, diculairement à un champ de radiofréquence $H_{1} \cos \omega t$ parallèle à $O z$. Lorsqu'on balaie un champ magnétique $H_{0}$ autour de zéro, parallèlement à $O z$, la modulation à la fréquence $\omega / 2 \pi$ de la lumière absorbée, recueillie par le photomultiplicateur P.M., subit, comme il a été indiqué dans la communication précédente, une variation résonnante en forme de courbe de dispersion ( $f i g .2$ ). Ainsi à toute variation $\delta H_{0}$ autour

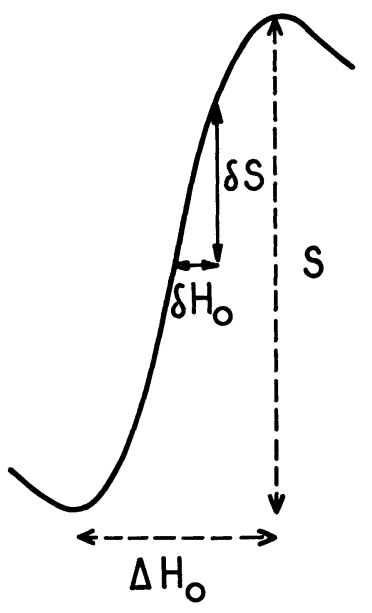

FIG. 2.

de zéro de la composante le long de $O z$ du champ magnétique, correspond une variation $\delta S$ du signal détecté qui fournit ainsi une mesure de $\delta H_{0}$. Il est évident qu'on ne peut ainsi mesurer que des variations de champs inférieures à la largeur en champ $\Delta H_{0}$ de la courbe de dispersion utilisée, variations qui sont donc en fait limitées à un intervalle de l'ordre du $\mu \mathrm{G}$, 
pour lequel la correspondance entre $\delta S$ et $\delta H_{0}$ est pratiquement linéaire.

On réalise ainsi un magnétomètre en champ très faible permettant de mesurer sélectivement la composante du champ magnétique parallèle à une direction donnée $(\mathrm{Oz})$. En effet, comme nous l'avons vu dans la communication précédente, le signal détecté $\delta S$ peut se mettre pour des variations faibles du champ magnétique sous la forme du développement en série :

$$
\begin{aligned}
\delta S=\text { Cte } & . M_{0}^{\prime} J_{0}\left(\frac{\omega_{1}}{\omega}\right) J_{1}\left(\frac{\omega_{1}}{\omega}\right) \sin \omega t\left[\gamma \delta H_{z} \tau\right. \\
& \left.-J_{0}^{2}\left(\frac{\omega_{1}}{\omega}\right)\left(\gamma \delta H_{x} \tau\right)\left(\gamma \delta H_{y} \tau\right)\right]+\ldots
\end{aligned}
$$

et se trouve donc insensible au premier ordre aux composantes $\delta H_{x}$ et $\delta H_{y}$ du champ magnétique perpendiculaire à $O z$.

B. Description du montage expérimental. - Il est clair que la sensibilité de ce magnétomètre nécessite une atténuation considérable des bruits magnétiques et des inhomogénéités statiques nécessairement présents dans un laboratoire et dont l'ordre de grandeur est le milligauss ou la fraction de milligauss. Aussi le dispositif expérimental se trouve-t-il protégé par un blindage magnétique de haute performance qui constitue un élément essentiel du montage et que nous allons commencer par décrire.

1. Le blindage magnétiQue. - Il se compose de cinq enceintes cylindriques concentriques de mu-métal de $2 \mathrm{~mm}$ d'épaisseur (longueur du cylindre intérieur : $1 \mathrm{~m}$, diamètre : $50 \mathrm{~cm}$ ). Chaque cylindre est fermé à ses deux extrémités par des couvercles de mu-métal de même épaisseur percés de fenêtres permettant aux faisceaux de pompage et de détection de traverser le blindage.

Le facteur de blindage des perturbations magnétiques dans la direction perpendiculaire à l'axe des cylindres est supérieur à $10^{5}$, ce qui permet de réduire le bruit magnétique dans cette direction nettement au-dessous de $10^{-9} \mathrm{G}$ lorsque les conditions magnétiques sont favorables dans le laboratoire (jours fériés ou nuit).

Le facteur de blindage est beaucoup moins bon dans la direction parallèle à l'axe des cylindres (de l'ordre de quelque $10^{4}$ ), mais le magnétomètre n'est pas sensible à la composante du champ dans cette direction qui est la direction $O x$ du faisceau de pompage.

La démagnétisation que l'on doit réaliser en principe après chaque « accident magnétique » survenu au blindage (variation brutale du champ auquel il a été soumis, ouverture et fermeture des extrémités, etc.) est assurée par un conducteur unique en cuivre, le traversant de part en part qui débite plusieurs centaines d'ampères de courant alternatif. On assure la démagnétisation en augmentant puis en diminuant progressivement l'intensité du courant, ce qui fait parcourir au mu-métal un très grand nombre de cycles d'hystérésis dont l'amplitude est d'abord croissante, puis décroissante.

Le champ résiduel à l'intérieur du blindage dépend précisément des conditions de la démagnétisation. Lorsque celle-ci a été soignée, ce champ peut être de l'ordre du $\mu \mathrm{G}$ (nous verrons plus loin comment on le mesure). Sa direction est arbitraire et dépend égale- ment des conditions de la démagnétisation. Des bobines de Helmholtz dans les trois directions sont prévues pour le compenser (voir paragraphe suivant).

Enfin, le blindage réduit également considérablement les inhomogénéités magnétiques sur le volume de la cellule de résonance (de l'ordre de $100 \mathrm{~cm}^{3}$ ). Celles-ci sont dans le laboratoire de l'ordre du milligauss et sont réduites au-dessous du $\mu \mathrm{G}$ dans le blindage.

Notons enfin qu'il est indispensable d'exclure du blindage tous les matériaux susceptibles d'être magnétiques et de créer des champs magnétiques et surtout des gradients de champ sur le volume de la cellule : les photomultiplicateurs (dont les dynodes sont en nickel), les circuits électroniques (dont les transistors sont magnétiques) et en général tous les matériaux pouvant comporter des impuretés magnétiques (laiton, etc.).

2. Le magnétomètre. - Il est schématisé sur la figure 3. La cellule de résonance, de $6 \mathrm{~cm}$ de diamètre, est située au centre du blindage. Sa paroi interne est enduite de paraffine et elle ne contient pas de gaz tampon.

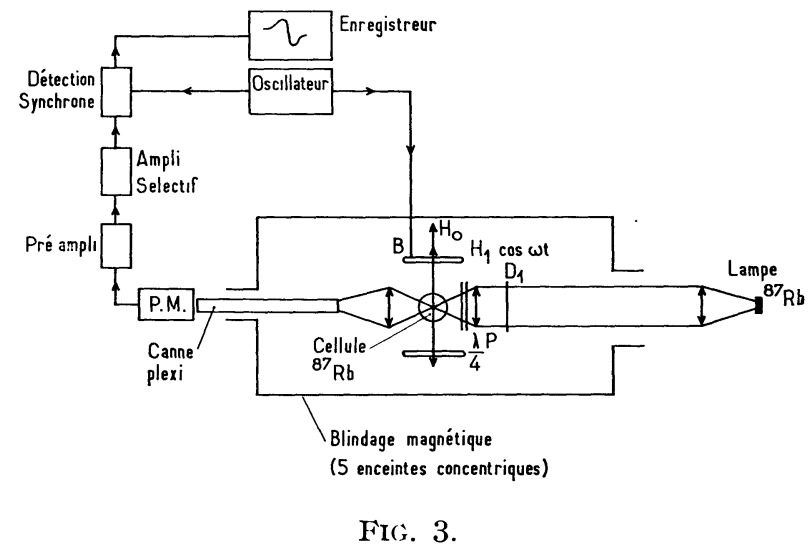

Le temps de relaxation $T$ des atomes de ${ }^{87} \mathrm{Rb}$ qu'elle contient est déterminé par les collisions sur la paroi paraffinée [4] et est de l'ordre de $1 \mathrm{~s}$.

D'autre part, les atomes moyennent par le mouvement les inhomogénéités magnétiques résiduelles dans le blindage qui ne sont donc pas une cause d'élargissement de la raie. La cellule est maintenue à la température ordinaire.

L'excitation optique est réalisée par une lampe à ${ }^{87} \mathrm{Rb}$ extérieure au blindage. Le faisceau de pompage $F$ pénètre dans le blindage par une fenêtre, est polarisé en $\sigma^{+}$et filtré (composante $D_{1}$ de la raie de résonance optique, $\lambda=7947 \AA$ ) à l'aide d'un filtre interférentiel. Après la traversée de la cellule, la lumière est recueillie par une canne en plexiglas qui la conduit hors du blindage jusqu'au photomultiplicateur à infrarouge (P.M. RGA 7102).

Le champ de radiofréquence, à $420 \mathrm{~Hz}$, est délivré par des bobines $B$ dont l'axe est parallèle à $O z$ et qui sont alimentées par un oscillateur Schlumberger Solartron.

Le signal convenablement préamplifié et amplifié sélectivement est analysé par une détection synchrone P.A.R. dont la constante de temps est réglée à $3 \mathrm{~s}$ et dont la sortie est reliée à un enregistreur Sefram. 
C. Compensation du champ résiduel à l'intérieur du blindage. - Il est nécessaire de compenser soigneusement, avant d'appliquer le champ à mesurer les trois composantes $\Delta H_{x}, \Delta H_{y}, \Delta H_{z}$ du champ magnétique résiduel à l'intérieur du blindage.

Les compensations sont assurées par un ensemble de trois paires de bobines de Helmholtz dont les axes sont respectivement parallèles aux trois directions $O x, O y$, $O z$. Ces bobines sont alimentées par des alimentations stabilisées en courant P.A.R. Comme elles doivent compenser des champs très faibles (de l'ordre de $1 \mu \mathrm{G}$ ), elles ne sont constituées que d'un seul tour de fil, les courants qui les parcourent étant de l'ordre de quelques $\mu \mathrm{A}$ (leur diamètre est de l'ordre de $20 \mathrm{~cm}$ ).

Remarquons également que, étant donné les très faibles champs qu'elles sont amenées à compenser, les inhomogénéités des champs qu'elles créent restent très faibles en valeur absolue et sont moyennées par le mouvement des atomes dans la cellule. C'est là un avantage supplémentaire du blindage, car s'il fallait compenser à l'aide de bobines le champ terrestre ambiant, les inhomogénéités dues aux bobines de compensation (ainsi d'ailleurs que les instabilités de courant) seraient prohibitives.

Afin de réaliser pratiquement la compensation du champ dans les trois directions, on se sert du signal de détection $\delta S$ donné par l'expression (1). On voit sur cette expression que le signal dépend au second ordre du produit $\Delta H_{x} . \Delta H_{y}$ des composantes résiduelles du champ perpendiculaires à $O z$. Ceci montre notamment que si $\Delta H_{x}$ (ou $\Delta H_{y}$ ) est nul, le signal devient insensible à $\Delta H_{y}$ (ou $\Delta H_{x}$ ). Cette constatation nous permet de régler à zéro la composante $\Delta H_{x}$ : il suffit de décompenser d'une quantité connue le champ le long de $O y$ et de recommencer l'expérience pour différentes valeurs de $\Delta H_{x}$ jusqu'au moment où le signal devient insensible aux variations de $\Delta H_{y}$ : le courant circulant dans les bobines de compensation le long de $O x$ est alors réglé à la bonne valeur. On règle de façon analogue le champ le long de $O y$. On annule ainsi les composantes du champ perpendiculaires à $O z$. Il ne reste plus alors qu'à annuler le signal $\delta S$, ce qui permet de compenser le champ dans la dernière direction. La mesure des courants nécessaires à ces compensations donne une mesure des trois composantes du champ résiduel à l'intérieur du blindage que l'on trouve en général être de l'ordre de $1 \mu \mathrm{G}$.

Remarque. - Le réglage précédent n'est valable que si les composantes $\Delta H_{x}, \Delta H_{y}, \Delta H_{z}$ du champ résiduel sont suffisamment faibles pour que le développement (1) soit valable. On effectue en général un préréglage grossier des champs en utilisant un signal moins sensible au champ, le signal d'effet Hanle ordinaire décrit dans la communication précédente.

Nous sommes maintenant en mesure de comprendre la nécessité de la compensation que nous venons de décrire en détail. Appelons en effet $\delta$ h le champ magnétique appliqué à la sonde du magnétomètre, dont nous cherchons à mesurer la composante $\delta h_{z}$. Si la composante résiduelle du champ $\Delta H_{z}$ est différente de zéro, nous ne nous trouvons plus au centre de la courbe de dispersion de la figure 2 et nous ne disposons plus $\mathrm{du}$ gain maximum sur le signal correspondant au champ $\delta h_{z}$ à mesurer. Si, en particulier, $\Delta H_{z}$ est supérieur à $\Delta H_{0}$, on se trouve à l'extérieur de la résonance et la sensibilité du dispositif aux variations de $\delta h_{z}$ devient faible ou négligeable.

D'autre part, s'il existait une composante incontrôlée $\Delta H_{x}$ du champ dans la direction $O x$ par exemple, la formule (1) nous montre qu'il apparaîtrait un signal parasite $\delta S^{\prime}$ :

$$
\delta S^{\prime} \propto M_{0}^{\prime} \sin \omega t J_{0}^{3}\left(\frac{\omega_{1}}{\omega}\right) J_{1}\left(\frac{\omega_{1}}{\omega}\right)\left(\gamma \Delta H_{x} \tau\right)\left(\gamma \delta h_{y} \tau\right)
$$

proportionnel à la composante transverse $\delta h_{y}$ le long de $O y$ du champ $\delta$ h appliqué. Afin d'éviter ces signaux parasites et de rendre le magnétomètre insensible aux composantes respectivement le long de $O y$ et de $O x$ du champ à mesurer, il est donc également indispensable de compenser $\Delta H_{x}$ et $\Delta H_{y}$.

D. Etude de la sensibilité du magnétomètre [2]. La sensibilité du magnétomètre dépend essentiellement de deux facteurs : la largeur $\Delta H_{0}$ et le rapport signal-bruit $\mathrm{S} / \mathrm{B}$ de la résonance détectée. La variation la plus petite de champ magnétique détectable $\varepsilon_{h}$ (correspondant à un signal égal au bruit) est en effet :

$$
\varepsilon_{h}=\frac{1}{2} \frac{\mathrm{B}}{\mathrm{S}} \Delta H_{0} \text {. }
$$

Nous allons étudier successivement les différents paramètres intervenant dans la formule (3) et indiquer comment on peut les optimiser.

1. OPTIMISATION DES PARAMÈTRES EXPÉRIMENTAUX. - a) La largeur de la résonance. - Elle est donnée par la formule (cf. communication précédente) :

$$
\Delta H_{0}=\frac{2}{\gamma \tau}=\frac{2}{\gamma}\left(\frac{1}{T}+\frac{1}{T_{p}}\right)=\frac{2}{\gamma}\left(\frac{1}{T}+\alpha I\right)
$$

$I$ est l'intensité lumineuse proportionnelle à $1 / T_{p}$ :

$$
1 / T_{p}=\alpha I \text {. }
$$

L'expression précédente ne serait valable en toute rigueur que si la relaxation de l'orientation de ${ }^{87} \mathrm{Rb}$ était décrite par une seule constante de temps $T$, comme on l'a supposé par souci de simplicité dans la communication précédente. En fait, l'existence du spin nucléaire complique beaucoup les processus de relaxation en faisant apparaître notamment plusieurs constantes de temps dans l'évolution des diverses observables [4], la largeur $\Delta H_{0}$ étant en fait une fonction de ces diverses constantes de temps. Nous n'entrons pas ici dans le détail de l'étude de la largeur de raie - qu'il est possible de faire - et nous admettrons la formule (4) dans laquelle $T$ représente une « pseudo-constante de temps » typiquement de l'ordre de la seconde.

Nous voyons immédiatement d'après (4) qu'afin d'avoir la résonance la plus fine possible, il faut avoir un rapport gyromagnétique $\gamma$ le plus grand possible, un temps de relaxation $T$ le plus long possible, une intensité lumineuse $I$ la plus faible possible.

Nous avons choisi le ${ }^{87} \mathrm{Rb}$ parce que, parmi les alcalins pompés usuellement, il a le plus grand rapport gyromagnétique $\left(\gamma=4,4 \times 10^{6} \mathrm{rad} / \mathrm{s} / \mathrm{G}\right)$.

Le temps de relaxation $T$ est considérablement allongé grâce à l'utilisation d'une cellule paraffinée $(T \simeq 1 \mathrm{~s})$. 


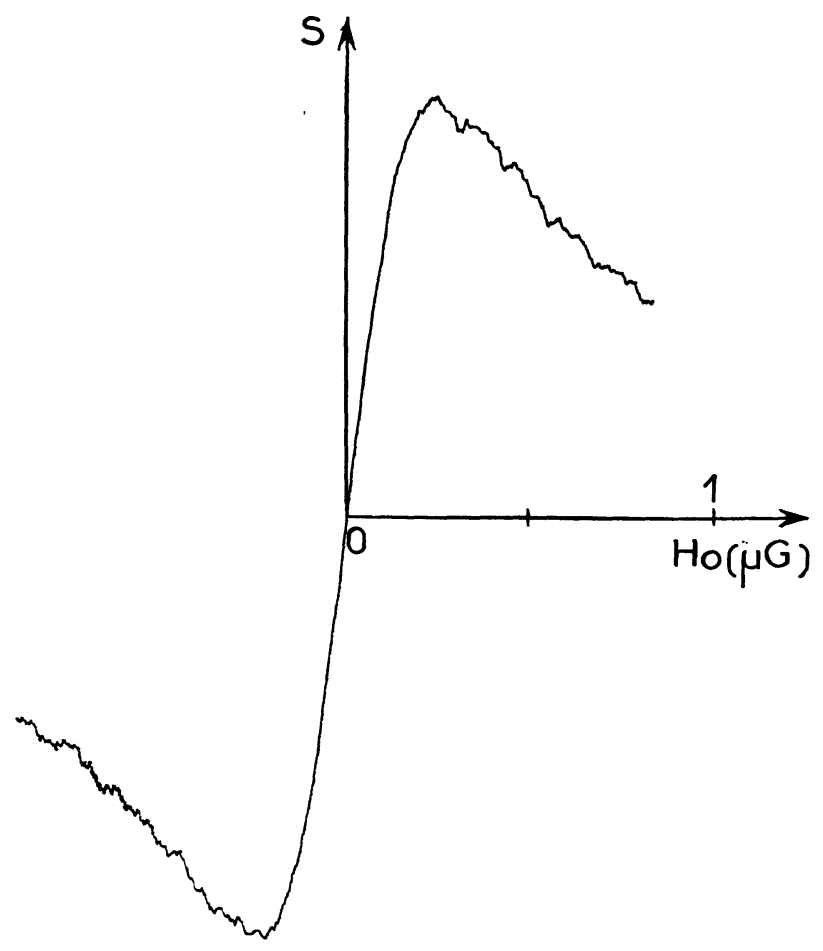

FIG. 4.

La figure 4 donne une idée de la finesse des résonances que l'on peut obtenir $\left(\Delta H_{0} \simeq 0,5 \mu \mathrm{G}\right)$ avec une intensité lumineuse $I$ très faible. Le rapport $\mathrm{S} / \mathrm{B}$ n'est pas excellent. Il dépend en effet également de $I$ et nous voyons ainsi qu'il est impossible de trop diminuer $I$, car cela s'effectue au détriment des autres paramètres qui régissent la sensibilité. Nous verrons plus loin $(\S \mathrm{d})$ comment on optimise l'intensité lumineuse.

Mentionnons pour finir d'autres facteurs d'élargissement de la raie non contenus dans (4), car ils sont précisément rendus négligeables ou très faibles dans les conditions expérimentales où l'on se place : les inhomogénéités magnétiques sont moyennées par le va-et-vient des atomes dans la cellule (il n'en serait plus de même si elle contenait un gaz tampon). Les collisions d'échange de spin [5] entre atomes dans la cellule ont un effet très faible sur la largeur de raie (contribution de l'ordre de $0,1 \mathrm{~Hz}$ ). Nous sommes en effet à température ordinaire (de l'ordre de $18{ }^{\circ} \mathrm{C}$ ); on peut montrer d'autre part qu'en champ $H_{0}$ très faible, les collisions d'échange n'affectent de toute façon que très peu la relaxation du moment transversal (si on appelle $T_{\mathrm{e}}$ le temps moyen séparant deux collisions d'échange, la relaxation n'affecte de façon importante le moment transversal que si le champ magnétique $H_{0}$ vérifie la condition $\gamma H_{0} T_{\mathrm{e}} \gg 1$; nous l'avons vérifié expérimentalement).

b) L'amplitude $S$ de la résonance. - La modulation $\omega / 2 \pi$ du moment transversal $M_{x}$ varie autour du champ nul suivant la relation (cf. communication précédente) :

$$
\begin{aligned}
& M_{x}(\omega)=M_{0}^{\prime} J_{0}\left(\frac{\omega_{1}}{\omega}\right) J_{1}\left(\frac{\omega_{1}}{\omega}\right) \sin \omega t \frac{\omega_{0} \tau}{1+\omega_{0}^{2} \tau^{2}} \\
& \text { avec : } \quad M_{0}^{\prime}=M_{0} \frac{1 / T_{p}}{\frac{1}{T_{p}}+\frac{1}{T}}=M_{0} \frac{\alpha I}{\alpha I+\frac{1}{T}}
\end{aligned}
$$

$M_{0}$ étant l'aimantation à saturation des atomes de ${ }^{87} \mathrm{Rb}$.
L'amplitude crête à crête de variation de cette modulation s'écrit donc :

$$
\begin{aligned}
\Delta M_{x}(\omega) & =M_{0}^{\prime} J_{0}\left(\frac{\omega_{1}}{\omega}\right) J_{1}\left(\frac{\omega_{1}}{\omega}\right) \\
& =M_{0} J_{0}\left(\frac{\omega_{1}}{\omega}\right) J_{1}\left(\frac{\omega_{1}}{\omega}\right) \frac{\alpha I}{\alpha I+\frac{1}{T}} .
\end{aligned}
$$

Le signal $S$ étant détecté sur la lumière du faisceau pompant est proportionnel à $I \Delta M_{x}(\omega)$, soit :

$$
S=K_{S} M_{0} J_{0}\left(\frac{\omega_{1}}{\omega}\right) J_{1}\left(\frac{\omega_{1}}{\omega}\right) \frac{\alpha I^{2}}{\alpha I+\frac{1}{T}}
$$

où $K_{S}$ est une constante de proportionnalité, dépendant notamment du nombre d'atomes $N$ présents dans la cellule.

La formule (8) nous montre que le signal est proportionnel au carré de l'intensité lumineuse $I$ et inversement proportionnel à la largeur de la résonance

$$
\Delta H_{0}=(2 / \gamma)\left(\alpha I+\frac{1}{T}\right)
$$

Afin d'augmenter le signal, on a intérêt à augmenter I. La largeur et le bruit augmentent alors corrélativement.

Laissant au $\S \mathrm{d}$ la discussion relative à l'intensité lumineuse, cherchons à optimiser les autres paramètres dont dépend $S:$ On a intérêt à minimiser $1 / T$, donc à allonger le temps de relaxation $T$ (cf. paragraphe précédent). On doit également régler l'amplitude $H_{1}$ du champ de radiofréquence de façon que le coefficient $J_{0}\left(\omega_{1} / \omega\right) J_{1}\left(\omega_{1} / \omega\right)$ soit maximum. Cela est réalisé pour $\omega_{1} / \omega \simeq 1,1$ et on a alors $J_{0}\left(\omega_{1} / \omega\right) J_{1}\left(\omega_{1} / \omega\right) \simeq 0,34$.

Notons que, dans ces conditions, le signal est stationnaire pour de petites variations de l'amplitude ou de la fréquence du champ de radiofréquence, ce qui élimine une cause d'instabilité du signal.

c) Le bruit. - Nous diviserons les différentes causes de bruit possibles en deux catégories suivant qu'elles sont d'origine magnétique ou liées au détecteur utilisé

Le bruit magnétique peut être dû soit aux fluctuations magnétiques résiduelles à l'intérieur du blindage $\left(<10^{-9} \mathrm{G}\right.$ dans la direction où le magnétomètre est sensible), soit aux fluctuations d'intensité des alimentations de compensation. Dans les deux cas, ce bruit se trouve nettement inférieur aux autres causes de bruit dans les conditions où nous avons travaillé. Il est facile de s'en rendre compte en comparant le niveau du bruit sur deux enregistrements, l'un effectué en champ nul, au centre de la résonance dans des conditions où la sensibilité magnétique du magnétomètre est maximum, l'autre dans un champ $H_{0} \gg \Delta H_{0}$ à l'extérieur de la résonance, dans des conditions où la sensibilité magnétique du dispositif est nulle. Le bruit est le même dans les deux cas, ce qui montre bien qu'il n'est pas d'origine magnétique (notons toutefois que ceci n'est vrai que de nuit dans des conditions magnétiques particulièrement calmes; de jour, le bruit magnétique beaucoup plus important devient parfaitement visible).

Finalement, le bruit se réduit essentiellement au 
bruit shot du photomultiplicateur utilisé pour la détection :

$$
B=K_{B} \sqrt{\frac{I}{\tau_{\mathrm{D}}}}
$$

où $\tau_{\mathrm{D}}$ est la constante de temps de la détection synchrone et $K_{B}$ une constante. Nous avons vérifié expérimentalement la proportionnalité de $B$ à $\sqrt{I}$ et à $\sqrt{1 / \tau_{\mathrm{D}}}$. Les résultats expérimentaux que nous présentons plus loin sont en général relatifs à une constante de temps de détection de $3 \mathrm{~s}$. La constante de temps que l'on peut utiliser dépend évidemment de la vitesse de variation des champs que l'on détecte. Remarquons que, contrairement aux méthodes de détection par bobines d'induction, dont la sensibilité est d'autant plus grande que la variation du champ est plus rapide, le magnétomètre que nous décrivons est d'autant plus sensible que les variations de champ à détecter sont plus lentes.

d) Optimisation de l'intensité lumineuse. - Nous avons vu que les différents paramètres qui interviennent dans l'expression de la sensibilité ne sont pas indépendants mais sont liés entre eux par l'intermédiaire de l'intensité lumineuse $I$. Il est facile de voir dans ces conditions qu'il existe une intensité optimale $I_{0}$ du pompage.

Regroupant les relations (3), (4), (8) et (9), on a en effet :

$$
\varepsilon_{h}=\frac{1}{\gamma} \frac{K_{B}}{K_{S} \sqrt{\tau_{\mathrm{D}}}} \frac{1}{M_{0} J_{0} J_{1}} \frac{\left(\alpha I+\frac{1}{T}\right)^{2}}{\alpha I^{3 / 2}} .
$$

En dérivant (10) par rapport à $I$, on obtient la valeur $I_{0}$ de $I$ qui minimise $\varepsilon_{h}$ :

$$
\alpha I_{0}=3 / T \text {. }
$$

L'intensité $I_{0}$ de pompage optimale est donc celle qui correspond à une largeur de la résonance égale à quatre fois la largeur extrapolée à intensité lumineuse nulle. Nous avons vérifié ce résultat expérimentalement. Pour une cellule dont le « pseudo-temps » de relaxation

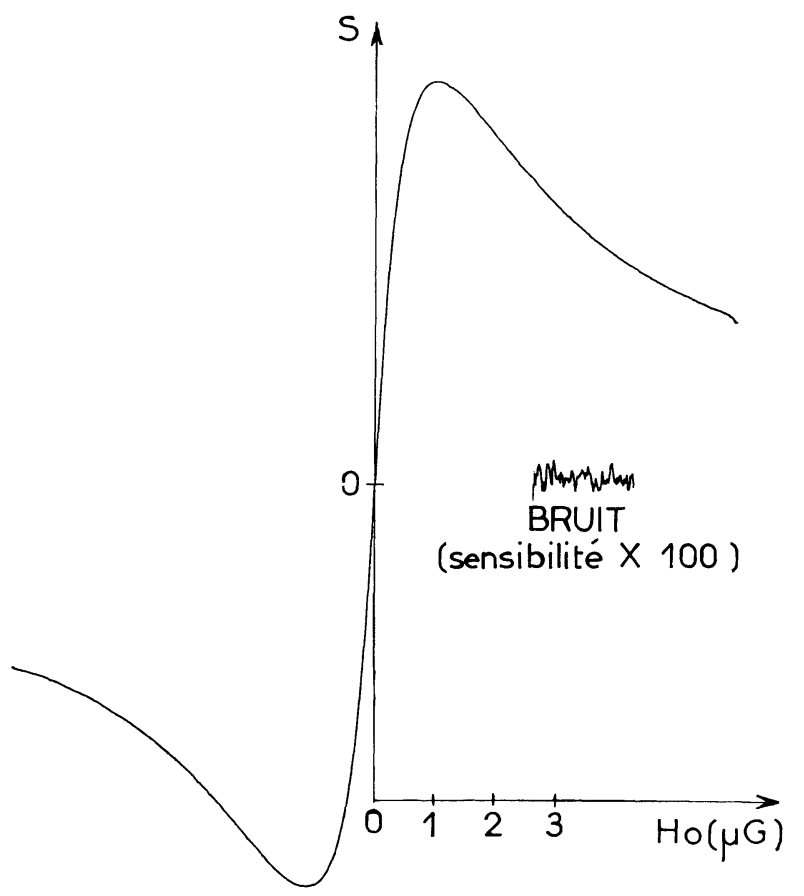

FIG. 5. est de l'ordre de $1 \mathrm{~s}$, on obtient ainsi une largeur optimale de l'ordre de $2 \mu \mathrm{G}$. La figure 5 montre la résonance obtenue dans ces conditions. La constante de temps de la détection synchrone est de $3 \mathrm{~s}$. Le niveau du bruit a été enregistré avec une amplification 100 fois plus grande que celle du signal. On voit que le rapport S/B est de l'ordre de 2500 . La sensibilité $\varepsilon_{h}$ attendue est alors, d'après (3) :

$$
\varepsilon_{h}=\frac{1}{2} \frac{2}{2500} \mu \mathrm{G} \simeq 4 \times 10^{-10} \mathrm{G} \text {. }
$$

2. Performances effectives du magnétomètre. Afin de tester effectivement la sensibilité du magnétomètre, on envoie dans une paire de bobines de Helmholtz dont l'axe est parallèle à $O z$ des créneaux de courant dont l'intensité correspond à une variation connue $\delta H_{0}$ du champ magnétique à mesurer et on enregistre la variation $\delta S \mathrm{du}$ signal correspondante. La figure 6 donne l'enregistrement obtenu pour des variations $\delta H_{0}=2 \times 10^{-9}$ G. La constante de temps de la détection synchrone est de $3 \mathrm{~s}$. On constate que la sensibilité du magnétomètre permet bien de détecter des variations de champ inférieures à $10^{-9} \mathrm{G}$.

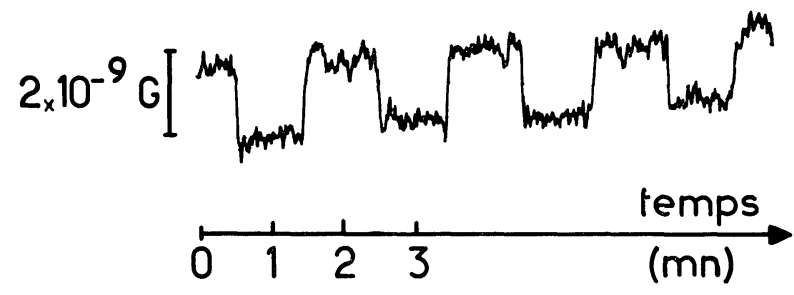

FIG. 6.

Si les variations du champ magnétique sont périodiques, il est possible d'améliorer encore la sensibilité du magnétomètre en utilisant les techniques de moyennage du bruit : la figure 7 donne un enregistrement sur

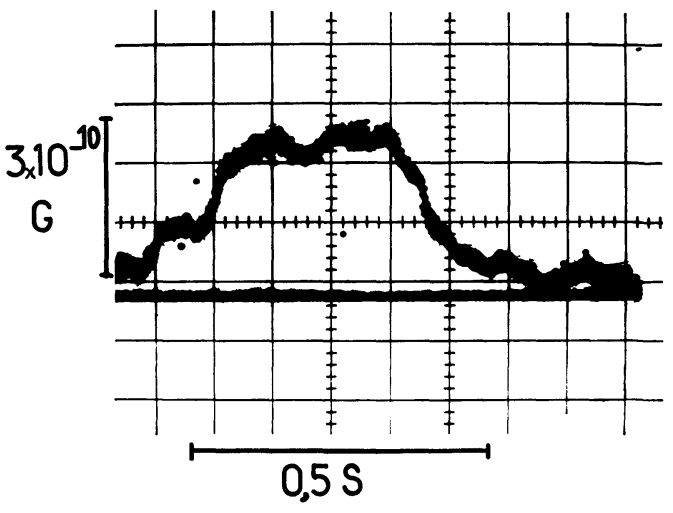

FIG. 7.

oscilloscope obtenu à la sortie d'un analyseur multicanaux, pour 3000 balayages successifs du champ à mesurer. La constante de temps de la détection synchrone a été réduite à $0,1 \mathrm{~s}$ afin de pouvoir accélérer la cadence de balayage sans déformer le signal. La constante de temps effective de la détection est donc de $300 \mathrm{~s}$, soit 100 fois plus longue que celle utilisée pour l'enregistrement de la figure 6 . On constate bien une amélioration de l'ordre de $\sqrt{100}=10$ du rapport $\mathrm{S} / \mathrm{B}$ : une variation de $10^{-10} \mathrm{G}$ devient à présent nettement appréciable. 
Il n'est pas sûr que les performances que nous venons de décrire constituent une limite des possibilités du dispositif, et des améliorations à sa sensibilité par perfectionnement du procédé de détection permettant d'augmenter le rapport $\mathrm{S} / \mathrm{B}$ sont actuellement à l'étude.

Nous venons donc de décrire un magnétomètre d'une très grande sensibilité, mais dont les possibilités semblent a priori limitées, car il ne permet de mesurer que des champs inférieurs au $\mu \mathrm{G}$.

En fait, il est possible de s'affranchir de ces limitations et de réaliser, sur les mêmes principes, un dispositif permettant de détecter, avec une sensibilité du même ordre, des champs magnétiques plus élevés.

Si le champ magnétique est supérieur à quelques $\mu \mathrm{G}$, on peut en effet utiliser, au lieu de la résonance apparaissant en champ nul, n'importe laquelle des résonances qui apparaissent pour les valeurs $H_{0}$ du champ magnétique telles que $\gamma H_{0}=n \omega$ et qui ont été décrites dans la communication précédente. Ces résonances ont la même largeur que la résonance $\omega_{0}=0$ et permettent donc en principe, autour du champ $H_{0}=n \omega / \gamma$, une détection aussi sensible du champ qu'autour du champ nul (en fait les signaux modulés résonnants pour $\omega_{0}=\gamma H_{0}=n \omega$ apparaissent sous forme d'une somme de signaux en absorption et en dispersion et afin d'isoler la composante en dispersion utile, il faut effectuer un réglage de phase délicat sur la détection synchrone).

E. Application du magnétomètre à la détection du champ magnétique statique créé par des noyaux de ${ }^{3} \mathrm{He}$ orientés par pompage optique [3]. - On peut envisager l'application du magnétomètre que nous venons de décrire à la détection du magnétisme de milieux très dilués. Nous l'avons effectivement utilisé pour la détection du champ magnétique statique créé par des noyaux de ${ }^{3} \mathrm{He}$ orientés par pompage optique en phase gazeuse. Remarquons que nous envisageons un cas qui semble particulièrement défavorable puisque nous cherchons à détecter le paramagnétisme d'origine nucléaire d'un milieu gazeux sous faible pression :

Soit une cellule sphérique contenant de l'hélium 3 sous une pression de $3 \mathrm{~mm} \mathrm{Hg}$. Nous pouvons orienter les noyaux de ${ }^{3} \mathrm{He}$ de spin nucléaire $I=1 / 2$ par le procédé classique du pompage optique de l'hélium [6]. Il est facile de calculer le champ magnétique créé à l'extérieur de la cellule par les noyaux ainsi orientés. Ce champ est le même que si tous les noyaux étaient localisés au centre de la cellule et est donc identique à celui d'un dipôle. Dans la direction parallèle à l'aimantation du milieu, ce champ est radial et vaut (en unités M.K.S.A.) :

$$
\Delta H=10^{-7}\left[\left(g \mu_{n}\right) / r^{3}\right] N P
$$

$r$ est la distance au centre de la cellule; $g \mu_{n} / 2$ est le moment magnétique nucléaire de ${ }^{3} \mathrm{He} ; N$, le nombre d'atomes contenus dans la cellule et $P$ le taux de polarisation. Avec une cellule de $6 \mathrm{~cm}$ de diamètre, une pression de $3 \mathrm{~mm} \mathrm{Hg}$, une polarisation de $5 \%$ (facilement réalisable), le champ à $6 \mathrm{~cm}$ du centre de la cellule est $\Delta H \simeq 5 \times 10^{-12}$ tesla $=5 \times 10^{-8} \mathrm{G}$. Quoique très faible, ce champ est nettement supérieur à la sensibilité ultime de notre magnétomètre, d'où l'idée de le mesurer effectivement et de se servir du magnétomètre pour une détection magnétostatique de la polarisation nucléaire de ${ }^{3} \mathrm{He}$.

Nous avons réalisé l'expérience dans les conditions

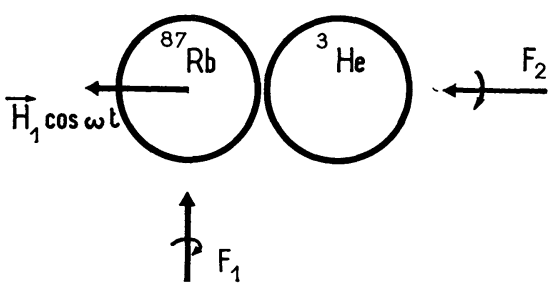

FIG. 8.

suivantes : Le montage expérimental est schématisé sur la figure 8 : une cellule de $6 \mathrm{~cm}$ de diamètre contient les noyaux de ${ }^{3} \mathrm{He}$; le champ magnétique qu'ils créent est détecté par les atomes de ${ }^{87} \mathrm{Rb}$ contenus dans la seconde cellule (l'ensemble des deux cellules est évidemment toujours placé dans le blindage magnétique précédemment décrit).

La partie «magnétomètre » du montage est identique à celle que nous avons décrite $\mathrm{au} \S \mathrm{B}:$ La cellule à ${ }^{87} \mathrm{Rb}$ est pompée par le faisceau $F_{1}$ perpendiculairement au champ de radiofréquence $H_{1} \cos \omega t$. Les atomes de ${ }^{3} \mathrm{He}$ sont pompés optiquement par un faisceau $F_{2}$ polarisé circulairement et se propageant parallèlement à $H_{1} \cos \omega t$. (La cellule à ${ }^{3} \mathrm{He}$ est en fait soumise également à l'action d'une décharge. Le pompage de ${ }^{3} \mathrm{He}$ est en effet plus compliqué que celui du rubidium et nécessite l'excitation intermédiaire - réalisée par la décharge - des atomes d'hélium dans un état métastable.) Quoi qu'il en soit, les spins nucléaires sont orientés en champ nul dans la direction de $F_{2}$, c'est-àdire dans celle de $H_{1} \cos \omega t$. La sonde à ${ }^{87} \mathrm{Rb}$ détecte la composante du champ dans cette direction qui se trouve être la composante radiale du champ dipolaire des noyaux de ${ }^{3} \mathrm{He}$. De plus, le champ magnétique créé par ${ }^{3} \mathrm{He}$ est moyenné par le mouvement des atomes de ${ }^{87} \mathrm{Rb}$; le magnétomètre détecte en fait la valeur moyenne de ce champ dans le volume de la cellule, c'est-à-dire finalement le champ en son centre qui est donné par la relation (12) où $r=6 \mathrm{~cm}$ représente la distance des centres des deux cellules.

La figure 9 montre la variation du champ magnétique détecté durant la transitoire de pompage optique de ${ }^{3} \mathrm{He}$. On constate que la variation du champ est bien de l'ordre de grandeur attendu. Le temps de pompage très long, de l'ordre de $10 \mathrm{mn}$, est caractéristique du pompage de l'hélium.

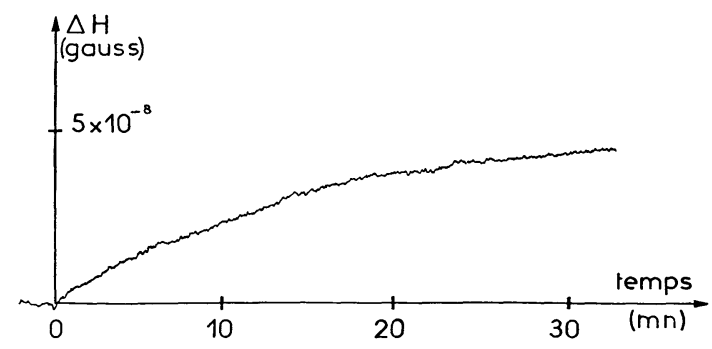

FIG. 9.

Nous avons également réalisé l'expérience suivante : Lorsque le taux de polarisation stationnaire est atteint, on interrompt le pompage optique de l'hélium et on éteint la décharge. On ajoute alors un champ magnétique $\boldsymbol{h}$ très petit, de l'ordre de $2 \mu \mathrm{G}$ perpendiculaire à la direction de pompage de l'hélium (ce champ étant donc perpendiculaire à $H_{1} \cos \omega t$ ne perturbe pas au 
premier ordre le signal détecté par le magnétomètre; il est de toute façon compensé au niveau de la sonde à ${ }^{87} \mathrm{Rb}$ par un jeu de bobines de Helmholtz supplémentaire).

Les noyaux d'hélium, initialement orientés le long de $H_{1} \cos \omega t$, se mettent à précesser autour de $\mathbf{h}$ à une fréquence de Larmor très petite $\nu\left(\nu \sim 6 \times 10^{-3} \mathrm{~Hz}\right)$. La composante radiale du champ produit au centre de la cellule détectrice à ${ }^{87} \mathrm{Rb}$ varie comme $\Delta H \cos 2 \pi v t$ et se trouve donc modulée à la fréquence de Larmor des spins de ${ }^{3} \mathrm{He}$.

La figure 10 a montre l'enregistrement du signal correspondant. L'amplitude de la modulation, $2 \Delta \mathrm{H}$, est de l'ordre de $10^{-7} \mathrm{G}$. L'orientation met plusieurs heures à s'amortir, la relaxation n'étant due qu'aux collisions des atomes d'He contre les parois de la cellule (le faisceau et la décharge sont éteints) et le temps de relaxation correspondant est très long ( $2 \mathrm{~h} 20 \mathrm{mn})$. Les figures $10 \mathrm{~b}$ et 11 montrent le signal restant au bout de 3 et de $11 \mathrm{~h}$. Dans ce dernier cas, l'amplification a été augmentée et la constante de temps de la détection portée à $10 \mathrm{~s}$.

Une modulation du champ dont l'amplitude est de $10^{-9} \mathrm{G}$ est visible avec un rapport signal-bruit de l'ordre de 3 ou 4. Le signal que l'on détecte alors correspond à un taux de polarisation de $5 \times 10^{-4}$, le nombre de noyaux orientés n'étant que de $5 \times 10^{13}$ par $\mathrm{cm}^{3}$.

Le procédé de détection magnétostatique que nous venons de décrire se révèle ainsi très intéressant pour l'étude de la polarisation des noyaux d'hélium et pourrait avoir de nombreuses applications (réalisation de gyromètres à ${ }^{3} \mathrm{He}$ par exemple).

D'autres applications de ce magnétomètre à divers domaines impliquant la mesure de champs très faibles sont envisageables. Citons par exemple la physique du solide (supraconductivité, magnétisme), le bio-

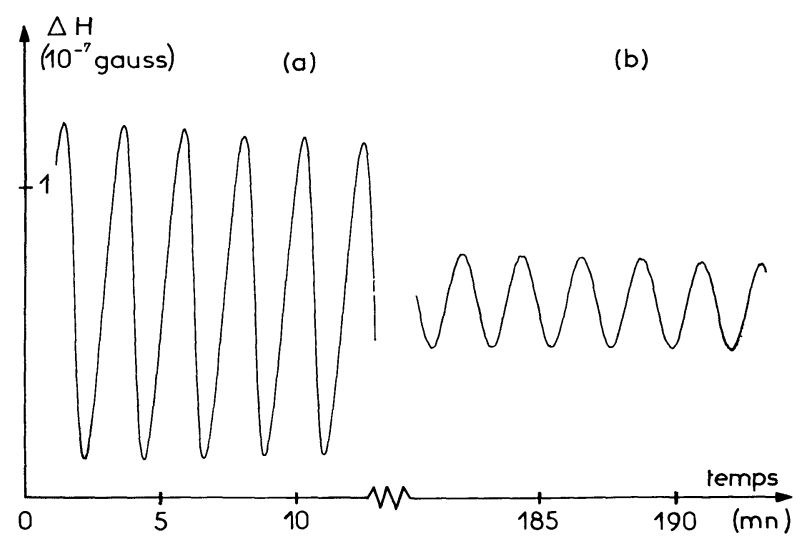

FIG. 10

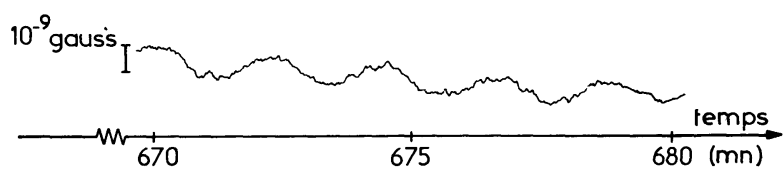

FIG. 11.

magnétisme (mesure des champs magnétiques biologiques), la physique spatiale (mesure des champs interplanétaires).

Signalons enfin une application possible du magnétomètre à l'asservissement des trois composantes d'un champ magnétique à la valeur zéro, avec une précision de l'ordre de $10^{-9} \mathrm{G}$. Nous avons vu en effet au $\S \mathrm{G}$ comment il était possible d'annuler dans les trois directions les composantes d'un champ résiduel. L'étude d'une compensation automatique du champ magnétique basé sur des méthodes analogues est actuellement en cours.

\section{BIBLIOGRAPHIE}

[1] La réalisation et les performances du magnétomètre que nous décrivons dans cette communication ont été rapportées dans la référence : DUPONT-Roc (J.), Haroche (S.) et Cohen-Tannoudji (C.), Phys. Letters, 1969, 28 A, 638.

Ce magnétomètre a fait, d'autre part, l'objet d'un brevet pris au nom du C.N.R.S. sous le numéro provisoire 177928, 1968.

[2] Pour une étude théorique de la sensibilité d'un magnétomètre à pompage optique d'une vapeur alcaline en champ très faible, voir : AlEXANDROV (E. B.), BONCH BRUEVICH (A. M.) et KHodovoy (B. A.), Opt. Spectry, 1967, 23, 151.
[3] Les expériences rapportées dans ce paragraphe on été décrites dans une lettre des auteurs à Phys. Rev. Letters : CohEn-Tannoudji (C.), DupontRoc (J.), Haroche (S.) et Lalö (F.), Phys. Rev. Lett., 1969, 22, 758.

[4] Bouchiat (M. A.) et Brossei (J.), Phys. Rev., 1966, 147, 41.

[5] Grossetête (F.), J. Physique, 1964, 25, 383.

[6] Colegrove (F. D.), Schearer (L. D.) et WaLTERS (G. K.), Phys. Rev., 1963, 132, 2567. 